\title{
Epidemiology and Antifungal Resistance in Invasive Aspergillosis aCCORDing to Primary Disease - REVIEW OF THE LITERATURE
}

\author{
A. Mayr, C. Lass-Flörl \\ Division of Hygiene and Medical Microbiology, Department of Hygiene, Microbiology and Social Medicine, \\ Innsbruck Medical University, Innsbruck, Austria
}

\begin{abstract}
Aspergilli, less susceptible to antifungals emerge and resistance to azoles have been found mainly in $A s$ pergillus fumigatus; this has launched a new phase in handling aspergillosis. Resistant strains have currently been reported from Belgium, Canada, China, Denmark, France, Norway, Spain, Sweden, The Netherlands, UK and the USA. Centres in the UK (Manchester) and The Netherlands (Nijmegen) have described particularly high frequencies (15 and 10\% respectively), and a significant increase in azole resistance in recent years. The reason of this high incidence may be due to long term azole therapy in patients with chronic aspergillosis in Manchester, and due to high use of agricultural azoles in Nijmegen. The primary underlying mechanism of resistance is as a result of alterations in the cyp5 $1 \mathrm{~A}$ target gene, with a variety of mutations found in clinical isolates and one genotype identified in the environmental (LH98). Reports on well documented in vitro and in vivo resistance to echinocandins are rare for Aspergillus species and resistance may be under-diagnosed as susceptibility testing is less frequently performed due to technical reasons.
\end{abstract}

Key words: Epidemiology, antifungal resistance, $A s$ pergillus, azoles, candins

\section{INTRODUCTION}

Invasive fungal infections (IFD) are increasingly recognized and represent a primary cause of morbidity and mortality in critically ill patients [1-4]. A variety of factors, including immunosuppressive agents, broadspectrum antibiotics, and antineoplastic agents influence the incidence and severity of IFDs [1]. Transplant and haematopoietic stem cell transplant recipients, intensive care unit and surgical patients display the population at risk [1, 4-7]. Aspergillus species have become the most important pathogens [8]. The introduction of voriconazole, posaconazole and echinocandins (caspofungin, micafungin and anidulafungin) improved the therapeutic option for treatment of invasive aspergillosis (IA) [9]. Although the outcome of IA is largely influenced by the state of immunosuppression, factors related to the fungus also play a role. Un- til recently, species identification was sufficient to guide antifungal therapy, but the emergence of acquired resistance limits the use of species identification for predicting activity of antifungal agents. Aspergilli, less susceptible to antifungals emerged and acquired resistance to azoles have been found mainly in Aspergillus fumigatus [10]; this has launched a new era in handling aspergillosis. This article reviews the epidemiology and antifungal resistance against azoles and candins with particular emphasis on Aspergillus species.

\section{EPIDEMIOLOGY of INVASIVE AsPeRgILLOSIS}

In a 4-year-study Pagano et al. [11] showed that 64\% of IFDs in patients with haematological malignancies were caused by moulds and among them 90\% were due to Aspergillus species. Overall, the incidence of IA varies according to underlying diseases, pathogen [1f] and geographic location [10, 12, 13]; rates of up to $7 \%$ are reported in Europe [11]. Mortality rates for IA are high and vary according to patient population, ranging from $38 \%$ in patients with acute myelogenous leukaemia, from $50-60 \%$ in patients with organ transplantation and from $70-85 \%$ in other immunosuppressed patients [11-16].

Although $A$. fumigatus still represents the leading cause of IA, species like Aspergillus terreus and $A$ spergillus flavus become more frequent $[15,17]$. These non- $A$. fumigatus may be intrinsically resistant to antifungal agents (eg A. ustus) [18] and the clinical presentation and evolution of IA may differ from commonly observed $A$. fumigatus infections [18-23]. Maximizing the efforts to culture the causative pathogen appears necessary to allow identification and susceptibility testing.

\section{Epidemiology of Azole And Candine Resistance in Clinical $A$. FUMigatus}

Antifungal drug resistance is normally quantified using the minimum inhibitory concentration (MIC). The MIC represents the lowest drug concentration that results in a notably reduction or complete lack of fungal growth. In vitro resistance can be primary (intrinsic) or secondary (acquired). Primary resistance occurs 
Table 1. Azole resistance in A. fumigatus.

\begin{tabular}{ll}
\hline Phenotypes & Description \\
Pan-azole resistant & MICs are in the resistant range for all available active azoles \\
Multi azole resistance & MICs are in the resistant range for more than one, but not all azoles \\
Itraconazole, voriconazole or posaconazole resistant & MICs are in the resistant range for a single azole \\
\hline
\end{tabular}

MICs $=$ minimal inhibitory concentrations

Table 2. Clinical overview of azole resistance in $A$. fumigatus.

\begin{tabular}{llll}
\hline Location & Clinical presentation & Epidemiology & Pathomechanism \\
\hline Nijmegen & Invasive pulmonary aspergillosis & Mainly azole naive & Dominant resistance \\
& CNS aspergillosis & Previous azole & mechanism: \\
& Osteomyelitis & exposure & TR/L98H \\
Manchester & Chronic aspergillosis & Previous azole & Multiple resistance \\
& Aspergilloma & exposure & mechanisms: \\
& ABPA & & G448S, M220, P216L, \\
& CNS aspergillosis & TR/L98H \\
\hline
\end{tabular}

$\mathrm{ABPA}=$ allergic bronchopulmonary aspergillosis

naturally, without prior exposure to the drug. Secondary resistance is generated following exposure to an antifungal and may be associated with an altered gene expression $[10,24]$. Clinical resistance is defined as the failure to eradicate an infection despite the administration of an adequate antifungal [24]. Such failures can be attributed to a combination of the host, the pathogen and the drug [24].

Using the European Committee for Antibiotic Susceptibility Testing (EUCAST) methodology breakpoints were recently proposed for $A$. fumigatus and itraconazole, voriconazole and posaconazole [25]; for itraconazole and voriconazole, $<2 \mathrm{mg} / \mathrm{L}$ (susceptible), $2 \mathrm{mg} / \mathrm{L}$ (intermediate) and $>2 \mathrm{mg} / \mathrm{L}$ (resistant); for posaconazole, $<0.25,0.5$ and $>0.5 \mathrm{mg} / \mathrm{L}$ respectively. It is suggested to differentiate between single-azole, pan-azole and multi-azole resistance (see Table 1), the majority of infections is associated with clinical failure when treated with the ascertained agent [26].

It was thought that acquired resistance of $A s$ pergillus species to triazoles is unfrequently [27, 28]. Yet reports from the Netherlands and Manchester display an alarming increase of azole resistance in $A$. $f u$ migatus since 1998 [26, 29, 30]. The first published case of itraconazole-resistance in $A$. fumigatus appeared in 1997 [31]; in 2000, a survey testing over 900 isolates showed a $2 \%$ prevalence of ITC resistance in Manchester [32]. In 2007 the percentage of patients with an azole-resistant $A$. fumigatus increased up to $15 \%[25,26]$. In the Netherlands azole resistance increased dramatically from $2.5 \%$ in 2000 , to $4.9 \%$ in 2002 , to $6.6 \%$ in 2004 and to $10 \%$ in 2009 [25]. This represents an increasing frequency of $6 \%$ per year and is an issue due to the limited number of antifungals. Overall, azole-resistance differs from country to country and occurred sporadically in Belgium, Denmark, France, Sweden, Spain and Norway [33-39]. In Spain, the prevalence is about $2 \%$ among clinical $A$. fumigatus and in Austria about $0 \%$. In the USA, species with
MICs of voriconazole and posaconazole $>2 \mathrm{mg} / \mathrm{L}$ remain rare, less than $1 \%$ [27].

The clinical presentation and disease evolution may be related to the underlying genotypes in $A$. fumigatus (Table 2). Isolates have been collected from patients suffering from chronic aspergillosis [29] and invasive diseases [26, 33].

Azole drug resistance in $A$. fumigatus has been reported both, before and after drug exposure; acquired resistance appears to develop through treatment of patients or through exposure of isolates to azole fungicides in the environment [40].

These findings have major implications for clinical practice especially as fungal drug resistance is an acute issue due to the limited number of antifungal compounds. Alternative strategies utilising combination therapy will become more attractive. Experts expect that triazole resistance in this haploid, sparingly sexual worldwide airborne fungus will increase [8]. Key elements in the management of patients will be an accurate speciation of Aspergillus species and the performance of in vitro susceptibility testing for an approbiate antifungal treatment. Presently we do not have exact data on the prevalence of azole-resistance Aspergilli in Germany, but seems to be rather low than high. Anyway, a shift has occurred in the epidemiology of invasive infections in Europe [8]. Where invasive candidiasis was once the predominant type of invasive fungal infections, invasive mould infections have become increasingly important, including those caused by unusual pathogens. Moulds have become the leading cause of IFD in some populations. Aspergillus species are the most frequent mould pathogens, but the number of infections caused by previously rare pathogens, such as the Zygomycetes and Fusarium species, is increasing. The reasons for the shift in the epidemiology are multifactorial, but are a result, at least in part, of the increased use of extensive voriconazole and echinocandins as prophylaxis/treat- 
ment [8]. As a result of growing numbers of immunosuppressed patients with risk factors, the patient populations for IA will expand and will include patients with haematological malignancy, ICU intervention, pulmonary disease [e.g.chronic obstructive pulmonary disease (COPD) and asthma], SOT recipients and patients with solid tumours.

Much less is currently known about echinocandin resistance in Aspergillus, in part because susceptibility testing is not routinely performed and because the methods suffer from technical difficulties and suboptimal reproducibility [41, 42]; breakthrough infections with $A$. fumigatus showing high minimum effective concentrations have been reported sporadically [43]. So far, the selection pressure of candins has risen and the development of resistance is presumed to be inevitable.

\section{MECHANism of AzOLE-RESISTANCE IN A. FUMIGATUS}

The triazoles block the ergosterol biosynthetic pathway at the C14- $\alpha$-demethylation stage [44]. These antifungals bind to lanosterol 14- $\alpha$-demethylase (14- $\alpha$ $\mathrm{DM}$, or Cyp51p) which is encoded by the Erg11 genes. Such step leads to depletion of ergosterol and an accumulation of lanosterol and other toxic 14- $\alpha$ methylated sterols.

Several pathomechanisms account for azole resistance in $A$. fumigatus; these include a modification of target enzymes, an increased expression of drug efflux mechanisms, an overexpression of target enzymes, an incorporation of exogenous cholesterol, an overexpression of HSP90 and of a sterole-regulatory element binding protein $[45,46]$. The resistance phenotype depends on the amino-acid substitution and more than one azole can be affected. Azole-resistant isolates have been reported as multidrug resistant [47], multiazole resistant [48], azole cross-resistant [27] and multiple-triazole resistant $[26,33]$ isolates. In most cases azole resistance has been associated with point mutations in $\operatorname{cyp} 51 \mathrm{~A}$, which represents the target enzyme of the azoles [25]; hot spots at codons 54, 98 and 220 are most frequently characterized [26, 33, 35, 48-50]. Interestingly, other mutations have been found in azole susceptible strains and so are unlikely to be associated with resistance [51].

The resistance mechanisms differ between the Dutch and British azole-resistant isolates; in the Netherlands, the presence of a single resistance mechanism (denoted by TR/L98H, a point mutation at codon 98 accompanied by a tandem repeat in the promoter region), was found in over $90 \%$ of clinical A. fumigatus isolates. By contrast, several CYP51 $A$ mutations are present in the UK strains [25, 33] and no prevalence of any one alteration. The reasons for this might be due to differences in the patient population from which the isolates originate [25]. For the Dutch isolates, an environmental source is very likely. Azole-resistance may develop due to exposure of $A$. fumigatus to azole fungicides for plant protection $[30,33]$. Howard et al. suggest that the reasons of the widespread increase of azole-resistance in the UK may be part of long-term azole drug exposures in patients [29].

\section{MECHANISM OF ECHINOCANDIN-RESISTANCE IN ASPERGILLUS SPEZIES}

The $\beta$-(1,3)-D-glucan is an integral part of the fungal cell wall. Echinocandins are a unique class which block the $\beta$ - $(1,3)-\mathrm{D}$-glucan synthesis by inhibiting $\beta$ - $(1,3)-\mathrm{D}$ glucan synthase [52]. This process leads to abnormal hyphal growth in moulds [24]. Much less is currently known about echinocandin resistance in Aspergillus and only few clinical isolates associated with treatment failures have been investigated. In such isolate mutation in the FKS 1 target gene was not detected, but expression of the FKS1 gene was found to be upregulated [37]. Manipulated or laboratory-selected strains with various degrees of caspofungin resistance have been described $[42,53,54]$. Some of these strains have been found to have mutations in the ECM33 gene (AfuEcm33), encoding cell wall proteins. Strains with mutations in the FKS1 gene encoding a subunit of the $\beta-1,3-D$-glucan synthase enzyme have been generated [42]. In other resistant Aspergillus mutants the glucan synthase exhibited a wild-type AfFKS1 gene sequence, where the function, level, and the enzyme itself were susceptible to caspofungin $[42,45]$.

Differences in the susceptibility to the echinocandins exist among the Aspergillus spezies. For example, Aspergillus niger is much more susceptible to echinocandins than other species probably in charge of its different cell-wall composition [55]. Aspergillus lentulus isolates are less susceptible to caspofungin, although they maintain susceptibility to anidulafungin and micafungin. The analysis of the A. lentulus fks sequence did not reveal a polymorphism at any of the known hot-spot regions of the gene [56].

\section{Cross Resistance Among Azoles And ECHINOCANDINS}

Cross-resistance patterns are closely linked with the position of the mutation in the cyp5 $1 A$ gene $[29,57]$. Isolates with alterations at eg codons 98 demonstrate a pan-azole resistance phenotype. Isolates with mutations at codon 54 remain voriconazole susceptible although cross-resistant to posaconazole.

Cross-resistance patterns in isolates with M220 alterations appear to be unpredictable, particularly with respect to voriconazole. The risk of cross-resistance between the azole compounds is high, in one report $74 \%$ and $65 \%$ of itraconazole resistant isolates were cross-resistant to posaconazole and voriconazole respectively [29].

Between itraconazole, voriconazole and ravuconazole cross-resistance was demonstrated in 10 clinical isolates of $A$. fumigatus obtained from patients with long-term exposure to itraconazole or voriconazole [50]. Also, broad-spectrum cross-resistance among all the azoles has been shown in $A$. fumigatus in a patient receiving prolonged itraconazole prophylaxis [47]. Overall, there is a limited number of reported cases that help us to understand the clinical impact of azole resistance on clinical outcome. For example, in a small case series of patients with IA and no respond to voriconazole, treatment with posaconazole was successful in 50\% of infections [58]. On the other hand 
in animal model of IA caused by an itraconazole-resis$\operatorname{tant} A$. fumigatus strain, posaconazole, which is structurally similar to itraconazole, was active in high doses [59].

The potential frequency of cross-resistance amongst echinocandins in Aspergillus species is still unclear and has not been investigated in detail [60]. At present, there is no evidence that the activity and efficacy of other antifungal compounds, such as the polyenes and echinocandins, is attenuated in azole-resistant isolates $[26,33]$.

\section{REFERENCES}

1. Holzheimer RG, Dralle H. Management of mycoses in surgical patients: review of the literature. Eur J Med Res 2002; 7: 200-26.

2. Jorda-Marcos R, Varez-Lerma F, Jurado M, et al. Risk factors for candidaemia in critically ill patients: a prospective surveillance study. Mycoses 2007; 50: 302-10.

3. Pfaller MA, Diekema DJ. Epidemiology of invasive candidiasis: a persistent public health problem. Clin Microbiol Rev 2007; 20: 133-63.

4. Mahfouz T, Anaissie E. Prevention of fungal infections in the immunocompromised host. Curr Opin Investig Drugs 2003; 4: 974-90.

5. Dimopoulos G, Piagnerelli M, Berre J, et al. Disseminated aspergillosis in intensive care unit patients: an autopsy study. J Chemother 2003; 15: 71-75.

6. Meersseman W, Lagrou K, Maertens J, et al. Invasive aspergillosis in the intensive care unit. Clin Infect Dis 2007; 45: 205-16.

7. Peres-Bota D, Rodriguez-Villalobos H, Dimopoulos G, et al. Potential risk factors for infection with Candida spp. in critically ill patients. Clin Microbiol Infect 2004; 10: 550 55.

8. Lass-Flörl C. The changing face of epidemiology of invasive fungal disease in Europe. Mycoses 2009: 1-9.

9. Walsh T, Annaissie E, Denning DW, et al. Treatment of Aspergillosis: Clinical Practice Guidelines of the Infectious Diseases Society of America. Clin Infect Dis 2009, 46: 327 60.

10. Lass-Flörl C. Azole Resistance in Aspergillosis: The Next Threat? Curr Fungal Infect Rep 2009; 3(4): 236-42.

11. Pagano L, Caira M, Candoni A, et al. The epidemiology of fungal infections in patients with hemotologic malignancies: the SEIFEM-2004 study. Haematologica 2006; 91: 1068-75.

12. Pagano L, Caira M, Nosari A, et al. Fungal infections in recipients of hematopoetic stem cell transplants: results of the SEIFEM B-2004 study - Sorveglianza Epidemiologica Infezioni Fungine Nelle Empatie Maligne. Clin Infect Dis 2007; 45: 1161-70.

13. Meersseman W, Vandecasteele SJ, Wilmer A, Verbeken E, Peetermans WE, Van Wijngaerden E. Invasive aspergillosis in critically ill patients without malignancy. Am J Respir Crit Care Med 2004; 170: 621-25.

14. Fourneret-Vivier A, Lebeau B, Mallaret MR, et al. Hospital-wide prospective mandatory surveillance of invasive aspergillosis in a French teaching hospital (2000-2002). J Hosp Infect 2006; 62: 22-28.

15. Lass-Flörl C, Grif K, Mayr A, et al. Epidemiology and outcome of infections due to Aspergillus terreus: 10-year single centre experience. Br J Haematol 2005; 131: 201-07.

16. Cornillet A, Camus C, Nimubona S, et al. Comparison of epidemiological, clinical, and biological features of invasive aspergillosis in neutropenic and nonneutropenic patients: a 6-year survey. Clin Infect Dis 2006; 43: 577-84.

17. Marr KA, Carter RA, Crippa F, et al. Epidemiology and outcome of mould infections in hematopoietic stem cell transplant recipients. Clin Infect Dis 2002; 34: 909-17.
18. Pavie J, Lacroix C, Hermoso DG, Robin M, Ferry C, Bergeron A, Feuilhade M, Dromer F, Gluckman E, Molina JM, Ribaud P. Breakthrough disseminated Aspergillus ustus infection in allogeneic hematopoietic stem cell transplant recipients receiving voriconazole or caspofungin prophylaxis. J Clin Microbiol 2005; 43: 4902-04.

19. Van der Linden J, Jansen R, Bresters D, et al. Azole-resistant central nervous system aspergillosis. Clin Infect Dis 2009, 48: 1111-13.

20. Steinbach WJ, Benjamin DK, Kontoyiannis DP, et al. Infections due to Aspergillus terreus: a multicenter retrospective analysis of 83 cases. Clin Infect Dis 2004; 39: 192-98.

21. Steinbach WJ, Perfect J, Schell WA, et al. In vitro analyses, animal models, and 60 clinical cases of invasive Aspergillus terreus infection. Antimicrob Agents Chemother 2004; 48: 3217-25.

22. Pfaller MA, Diekema DJ. Rare and emerging opportunistic fungal pathogens: concern for resistance beyond Candida albicans and Aspergillus fumigatus. J Clin Microbiol 2004; 42: 4419-31.

23. Balajee SA, Weaver M, Imhof A, et al. Aspergillus fumigatus variant with decreased susceptibility to multiple antifungals. Antimicrob Agents Chemother 2004; 48: $1197-$ 1203.

24. Kanafani ZA, Perfect JR. Resistance to Antifungal Agents: Mechanisms and Clinical Impact. Clin Inf Dis 2008; 46: 120-28.

25. Verweij PE, Howard S, Melchers WJG, et al. Azole-resistance in Aspergillus: Proposed nomenclature and breakpoints. Drug Resist Updat 2009; 12: 141-47.

26. Verweij PE, Melado E, Melcher WJ. Multiple-Triazole-Resistant Aspergillosis. N Engl J Med 2007; 356:1481-83.

27. Pfaller MA, Messer SA, Boyken L, et al. In vitro survey of triazole cross-resistance among more than 700 clinical isolates of Aspergillus species. J Clin Microbiol 2008; 46: 2568-72.

28. Guinea J, Recio S, Peáez T, et al. Clinical isolates of $A s$ pergillus species remain fully susceptible to voriconazole in the post-voriconazole era. Antimicrob Agents Chemother 2008; 52: 3444-46.

29. Howard SJ, Cesar D, Anderson MJ, et al. Frequency and evolution of azole resistance in Aspergillus fumigatus associated with treatment failure. Emerg Infect Dis 2009; 15: 1068-76.

30. Snelders E, Huis In't Veld RA, Rijs AJ, Kema GH, et al. Possible environmental origin of resistance of Aspergillus fumigatus to medical triazoles. Appl Environ Microbiol 2009; 75: 4053-57.

31. Denning D, Radford SA, Oakley KL, et al. Correlation between in-vitro susceptibility testing to itraconazole and invivo outcome of Aspergillus fumigatus infection. 1997, 40: 401-14.

32. Moore C, Walls C, Denning D. In vitro activity of the new triazole BMS-207147 against Aspergillus species in comparison with itraconazole and amphotericin B. Antimicrob Agents Chemother 2000, 44: 441-43.

33. Snelders E, van der Lee HAL, Kuijpers J, et al. Emergence of azole resistance in Aspergillus fumigatus and spread of a single resistance mechanism. PLoS Med 2008; 5: e219.

34. Van der Linden JWM, Snelders E, Debets Y, et al. Prospective study of the prevalence of azole resistance in Aspergillus species in the Netherlands. In: 48th Interscience Conference on Antimicrobial Agents and Chemotherapy, Washington 2008, abstract M-1718.

35. Mellado E, Garcia-Effron G, Alcazar-Fuoli L, et al. A new Aspergillus fumigatus resistance mechanism conferring in vitro cross-resistance to azole antifungals involves a combination of cyp51A alterations. Antimicrob Agents Chemother 2007, 51:1897-1904.

36. Lagrou K, De Vleeschouwer J, Meersseman W, et al. Triazole resistance among 229 clinical Aspergillus fumigatus isolates. In: $3^{\text {rd }}$ Advances Against Aspergillosis, Miami 2008, Abstract 33. 
37. Arendrup MC, Perkhofer S, Howard SJ, et al. Establishing in vitro-in vivo correlations for Aspergillus fumigatus: the challenge of azoles versus echinocandins. Antimicrob Agents Chemother 2008; 52: 3504-511.

38. Chryssanthou E. In vitro susceptibility of respiratory isolates of Aspergillus species to itraconazole and amphotericin B acquired resistance to itraconazole. Scand J Infect Dis 1997, 29:509-12.

39. Dannaoui E, Borel E, Monier M, et al. Acquired itraconazole resistance in Aspergillus fumigatus. J Antimicrob Chemother 2001, 47: 333-40.

40. Mortensen KL, Mellado E, Lass-Flörl C, et al. Environmental study of azole-resistant Aspergillus fumigatus and other aspergilli in Austria, Denmark, and Spain. Antimicrob Agents Chemother 2010; 54(11): 4545-9.

41. Arendrup MC, Garcia-Effron G, Buzina W, et al. Breakthrough Aspergillus fumigatus and Candida albicans Double Infection during Caspofungin Treatment: Laboratory Characteristics and Implication for Susceptibility Testing. Antimicrob Agents Chemother 2009; 53: 1185-93.

42. Gardiner RE, Souteropoulos P, Park S, et al. Characterization of Aspergillus fumigatus mutants with reduced susceptibility to caspofungin. Med Mycol 2005; 43(Suppl. 1): 299305.

43. Madureira A, Bergeron A, Lacroix C, et al. Breakthrough invasive aspergillosis in allogeneic haematopoietic stem cell transplant recipients treated with caspofungin. Int J Antimicrob Agents 2007; 30: 551-54.

44. Odds FC, Brown AJ, Gow NA. Antifungal agents: mechanisms of action. Trends Micrbiol 2003; 11: 279-99.

45. Chamilos G, Kontoyiannis DP. Update on antifungal drug resistance mechanisms of Aspergillus fumigatus. Drug Resist Updat 2005; 8: 344-58.

46. Gulshan K, Moye-Rowley W. Multidrug resistance in fungi. Eukaryot Cell 2007; 6: 1933-42.

47. Warris A, Weemaes CM, Verweij PE. Multidrug resistance in Aspergillus fumigatus. N Engl J Med 2002; 347: 2173-74.

48. Howard S, Webster I, Moore BC, et al. Multi-azole resistance in Aspergillus fumigatus. Int $\mathrm{J}$ Antimicrob Agents 2006, 28:450-53.

49. Chen J, Li R, Bu D, et al. Mutations in the cyp51A gene and susceptibility to itraconazole in Aspergillus fumigatus serially isolated from a patient with lung aspergilloma. J Antimicrob Chemother 2005; 55: 31-7.

50. Mellado E, Garcia-Effron G, Buitrago MJ, et al. Targeted gene disruption of the 14-\{alpha\} sterol demethylase (cyp51A) in Aspergillus fumigatus and its role in azole drug susceptibility. Antimicrob Agents Chemother 2005, 49: 2536-38.
51. Howard S, Arendrup MC. Acquired antifungal drug resistance in Aspergillus fumigatus: epidemiology and detection. Med Mycol 2010; 1-6.

52. Kartsonis N, Nielsen J, Douglas CM. Caspofungin: the first in a new class of antifungal agents. Drug Resist Updat 2003; 6: 197-218.

53. Romano J, Nimrod G, Ben-Tal N, et al. Disruption of the Aspergillus fumigatus ECM33 homologue results in rapid conidial germination, antifungal resistance and hypervirulence. Microbiology 2006; 152: 1919-28.

54. Perlin DS. Resistance to echinocandin-class antifungal drugs. Drug Resist Updat 2007; 10: 121-30.

55. Imhof A, Balajee SA, Marr KA. New methods to assess susceptibilities of Aspergillus isolates to caspofungin. J Clin Microbiol; 41: 5683-88.

56. Staab JF, Kahn JN, Marr KA. Differential Aspergillus lentulus echinocandin susceptibilities are Fksp independent. Antimicrob Agents Chemother 2010; 54(12): 4992-8.

57. Rodriguez-Tudela JL, Alcazar-Fuoli L, Mellado E, et al. Epidemiological cutoffs and cross-resistance to azole drugs in Aspergillus fumigatus. Antimicrob Agents Chemother 2008; 52: 2468-72.

58. Herbrecht R, Denning D, Patterson TF, et al. Voriconazole versus amphotericin $\mathrm{B}$ for primary therapy of invasive aspergillosis. N Engl J Med 2002, 347: 408-15.

59. Oakley KL, Morrissey G, Denning DW. Efficacy of SCH56592 in a temporarily neutropenic murine model of invasive aspergillosis with an itraconazole-susceptible and an itraconazole-resistant isolate of Aspergillus fumigatus. Antimicrob Agents Chemother 1997, 41: 1504-07.

60. Bal AM. The echinocandins: three useful choices or three too many? Int J Antimicrob Agents 2010; 35: 13-18.

Received: February 3, 2011 / Accepted: February 22, 2011

Address for correspondence:

Cornelia Lass-Flörl

Fritz Pregl Str 3/III

Department of Hygiene, Microbiology and Social Medicine

Division of Hygiene and Medical Microbiology

Innsbruck Medical University

Innsbruck

Austria

Tel.: +435129003 70703

Fax: $\quad+43512900373700$

E-mail: cornelia.lass-floerl@i-med.ac.at 